\title{
The prevalence of, and risk factors for, Barrett's oesophagus after sleeve gastrectomy
}

\author{
Marcin Migaczewski, Agata Czerwińska, Mateusz Rubinkiewicz, Piotr Zarzycki, Magdalena Pisarska, \\ Justyna Rymarowicz, Michał Pędziwiatr, Piotr Major \\ $2^{\text {nd }}$ Department of General Surgery, Jagiellonian University Collegium Medicum, Krakow, Poland
}

Videosurgery Miniinv 2021; 16 (4): 710-714

DOI: https://doi.org/10.5114/wiitm.2021.107776

\begin{abstract}
Introduction: Sleeve gastrectomy has become one of the most performed bariatric procedures. There is increasing evidence that sleeve gastrectomy patients suffer from gastroesophageal reflux disease and its sequelae such as erosive oesophagitis (EO) or Barret's oesophagus (BO).

Aim: To evaluate the incidence of $E O$ and $B O$ among patients with normal preoperative oesophagogastroduodenoscopy findings, who underwent sleeve gastrectomy, and investigate factors that may contribute to the development of $E O$ and $B O$ after the surgery.

Material and methods: We conducted a single-centre longitudinal study that included patients who underwent sleeve gastrectomy and completed 5 years of follow-up. Gastroscopies were performed in all patients at the end of the follow-up period. Patient- and treatment-related factors were used to search for risk factors of $B O$.

Results: From a total of 30 patients, symptomatic reflux was reported by 17 (56.7\%) during the follow-up period. At EGD EO was found in 9 of those patients (30\%), whereas BE was diagnosed in 8 (27\%) patients. The median BMI at the end of the follow-up period was significantly higher among patients with $B E$ than in the groups with EO and with no endoscopic changes: $40.91 \pm 6.32,32.42 \pm 5.53$, and $33.25 \pm 4.41$, respectively $(p=0.04)$.

Conclusions: The prevalence of $B O$ in $S G$ patients is considerable. The risk of $B E$ increases in patients with poor bariatric outcome. Endoscopic surveillance should be considered as part of the follow-up, especially in patients with higher overall risk of $B E$.
\end{abstract}

Key words: endoscopy, Barrett's oesophagus, bariatric procedures.

\section{Introduction}

The continuously rising obesity rate in western countries generates a substantial number of people requiring bariatric surgery. Sleeve gastrectomy (SG) has become one of the most frequently performed bariatric procedures globally. According to the International Federation for the Surgery of Obesity and Metabolic Disorders (IFSO), it accounted for nearly $44 \%$ of all primary bariatric procedures performed worldwide between 2013 and 2017 [1]. In countries, where the prevalence of obesity is particularly high, such us the United States, SG is performed even more often [2]. SG gained in popularity mainly due to its relative simplicity in comparison with other bariatric procedures, with similar long-term outcomes in weight reduction. Compared to Roux-en-Y gastric bypass, it is technically more feasible to perform and at the same time has fewer postoperative complications. Because there is no risk of internal hernias due to the lack of small bowel rearrangement, it also enables endoscopic access to an intact intestinal passage.

\footnotetext{
Address for correspondence

Marcin Migaczewski PhD, $2^{\text {nd }}$ Department of General Surgery, Jagiellonian University Collegium Medicum, Krakow, Poland,

phone: +48 1242482 00, e-mail: marcinmigaczewski@onet.pl
} 
However, there is increasing evidence that SG causes new-onset gastroesophageal reflux disease (GERD) or worsens pre-existing reflux symptoms [3-5]. In addition, morbidly obese individuals are affected by GERD symptoms more often than the general population. It has been postulated that elevated intra-abdominal pressure in obese individuals promotes GERD by disrupting the normal gastroesophageal pressure gradient [6]. GERD is a primary risk factor for erosive oesophagitis (EO) and Barrett's oesophagus (BO). Furthermore, several studies indicate that visceral adiposity and metabolic syndrome, which are frequently present in patients undergoing bariatric procedures, contribute to the development of $B O$ regardless of GERD $[7,8]$.

The majority of research studying the relationship between SG and GERD is based on reflux symptoms rather than endoscopic evaluation; therefore, data regarding the incidence rate of $\mathrm{EO}$ and $\mathrm{BO}$ among SG patients are limited $[9,10]$. Because the number of people undergoing SG is continuously growing, the prevalence of this important complication needs to be thoroughly investigated.

\section{Aim}

In our study we aim to evaluate the incidence of $\mathrm{EO}$ and $\mathrm{BO}$ among patients with normal preoperative oesophagogastroduodenoscopy (OGD) findings, who underwent SG, and investigate factors that may contribute to the development of EO and BO after the surgery.

\section{Material and methods}

The study group comprised 30 bariatric patients who underwent laparoscopic sleeve gastrectomy. Patients' inclusion criteria were as follow: age 18 years or older and younger than 60 years, and at least 5 years of follow-up. All patients fulfilled the criteria for bariatric surgery. Each had a body mass index $(\mathrm{BMI})>40 \mathrm{~kg} / \mathrm{m}^{2}$ or had a $\mathrm{BMI}>35 \mathrm{~kg} / \mathrm{m}^{2}$ with obesity-related comorbidities. In all patients an OGD was performed as part of the preoperative workup. All patients diagnosed with Helicobacter pylori underwent eradication prior to the surgery. All procedures were performed laparoscopically, and there was no need for conversion. Perioperative complications are reported using Clavien-Dindo classification. The routine follow-up appointments were arranged 10 days, 1 year, and after that every 3 years post index sur- gery. Endoscopic control was performed for every patient at the end of the follow-up period. BO was diagnosed according to the Seattle biopsy protocol (4-quadrant biopsy specimens taken at intervals of every 1 to $2 \mathrm{~cm}$ ) [11].

\section{Surgical technique}

Laparoscopic SG is perform using 5 trocars. The division of the vascular supply to the greater curvature of the stomach is performed with a radiofrequency device (Harmonic Scalpel ${ }^{\top M}$ ). A 36-Fr calibrating bougie is inserted alongside the lesser curvature, and a gastric tube is formed with a linear stapler. Running absorbable sutures are used to oversew the stapler line in order to decrease the risk of bleeding. Intra-abdominal drains are not routinely inserted.

\section{Ethical consideration}

The study was approved by the Bioethics Committee of the Jagiellonian University (122.6120.200.2016).

\section{Statistical analysis}

Statistica version 13 (StatSoft Inc., Tulsa, OK, USA) was used for data analysis. Variables are presented with median and range. Categorical data were compared using the $\chi^{2}$ test of independence. The Shapiro-Wilk test was used to check for normal distribution of the data. If the dataset had normal distribution the $t$ test was used, otherwise the Mann$U$-Whitney test was utilized. $P$-values $<0.05$ were considered statistically significant. All patient- and treatment-related factors were used to build a logistic regression model to search for risk factors of BO.

\section{Results}

Among the 30 patients included in the study, the follow-up time ranged between 65 and 123 months and ended in January 2020. The median maximal $\mathrm{BMI}$ of the study group was 47.78 (39.06-60.87) $\mathrm{kg} / \mathrm{m}^{2}$. Six patients suffered reflux symptoms preoperatively. However, the initial EGD findings were unremarkable in all patients with no signs of EO or BO.

During the postoperative period 1 patient suffered transient gastroparesis (Clavien-Dindo I), 2 patients had postoperative rhabdomyolysis, and 1 had dehydration requiring intravenous fluid resuscitation (Clavien-Dindo II). 
During the follow-up period symptomatic reflux was reported by 17 (56.7\%) patients. OGD with biopsy was performed in all patients in the study group. At OGD EO was found in 9 of those patients (30\%), whereas BO was diagnosed in $8(27 \%)$ patients. The remaining 13 (43\%) patients had no changes in pathological examination. The median initial BMI was 46.97 $\pm 7.52 \mathrm{~kg} / \mathrm{m}^{2}$ in the EO patients, $49.15 \pm 5.1 \mathrm{~kg} / \mathrm{m}^{2}$ in the BO patients, and $47.47 \pm 3.31 \mathrm{~kg} / \mathrm{m}^{2}$ in the group with no changes in endoscopy $(p=0.58, n s)$. Whereas at the end of the follow-up period the median BMI among patients with $\mathrm{BO}$ was significantly higher than in the groups with $\mathrm{EO}$ and with no endoscopic changes, $40.91 \pm 6.32 \mathrm{~kg} / \mathrm{m}^{2}, 32.42 \pm 5.53 \mathrm{~kg} / \mathrm{m}^{2}$, and 33.25 $\pm 4.41 \mathrm{~kg} / \mathrm{m}^{2}$, respectively $(p=0.04)$ (Table I).

Univariate logistic regression model revealed end $\mathrm{BMI}$ as a risk factor for $\mathrm{BO}$ occurrence in long term-observation $(\mathrm{OR}=1.373 ; 95 \% \mathrm{Cl}$ : 1.03-5.670; $p=0.013$ ). There was no statistically significant association between the likelihood of BO and demographic characteristics, obesity-associated comorbidities, or operating time (Table II).

Table I. Demographic characteristics and comorbidities of the 3 study groups

\begin{tabular}{|c|c|c|c|c|}
\hline Parameter & $\begin{array}{c}\text { No endoscopic } \\
\text { changes } \\
n=13\end{array}$ & $\begin{array}{l}\text { Erosive oesophagitis } \\
\qquad n=9\end{array}$ & $\begin{array}{l}\text { Barret's oesophagus } \\
\qquad n=8\end{array}$ & $P$-value \\
\hline Age & $38.6 \pm 9.56$ & $40.22 \pm 13.7$ & $41 \pm 12.3$ & 0.83 \\
\hline Gender (F/M) & $7 / 6$ & $1 / 8$ & $2 / 6$ & 0.36 \\
\hline ASA grade: & & & & 0.54 \\
\hline 1 & 0 & 1 & 0 & \\
\hline 2 & 11 & 6 & 7 & \\
\hline 3 & 2 & 1 & 1 & \\
\hline Maximal BMI & $47.47 \pm 3.31$ & $46.97 \pm 7.52$ & $49.15 \pm 5.1$ & 0.58 \\
\hline End BMI & $33.25 \pm 4.41$ & $32.42 \pm 5.53$ & $40.91 \pm 6.32$ & 0.04 \\
\hline Diabetes Mellitus & 1 & 2 & 1 & 0.64 \\
\hline Hyperlipidaemia & 6 & $5 / 2$ & $1 / 4$ & 0.35 \\
\hline Hepatosteatosis & 7 & 5 & 3 & 0.98 \\
\hline Hypertension & 7 & 6 & 6 & 0.63 \\
\hline Obstructive sleep apnoea & 3 & 3 & 2 & 0.87 \\
\hline Operating time & $151.15 \pm 35.7$ & $128.88 \pm 46.8$ & $125.62 \pm 39.04$ & 0.28 \\
\hline
\end{tabular}

Table II. Multivariate analysis of factors associated with Barrett's oesophagus after sleeve gastrectomy

\begin{tabular}{|lccc|}
\hline Parameter & OR & $95 \% \mathrm{Cl}$ & $P$-value \\
\hline Gender & 0.19 & $-3.479-0.163$ & 0.163 \\
\hline Age & 1.02 & $-0.052-0.092$ & 0.591 \\
\hline ASA grade & 6.483 & $-1.153-4,891$ & 0.225 \\
\hline Maximal BMI & 1.073 & $-0.088-0.229$ & 0.382 \\
\hline End BMI & 1.373 & $1.03-5.670$ & 0.013 \\
\hline Hyperlipidaemia & 1.154 & $-1.522-1.808$ & 0.866 \\
\hline Hepatosteatosis & 0.833 & $-1.803-1.438$ & 0.825 \\
\hline Hypertension & 2.077 & $-1.081-2.543$ & 0.43 \\
\hline Obstructive sleep apnoea & 0.889 & $-1.973-1.737$ & 0.901 \\
\hline Number of staples used & 1.341 & $-0.449-1.035$ & 0.439 \\
\hline Operating time & 0.989 & $-0.032-0.011$ & 0.326 \\
\hline
\end{tabular}




\section{Discussion}

Laparoscopic sleeve gastrectomy has become the most frequently performed bariatric procedure worldwide [1]. It has proven to be of equivalent effectiveness as the well-established Roux-en-Y gastric bypass, but it is technically easier to perform and provides endoscopic access to an intact intestinal passage [2, 12]. Several studies emphasized an augmented risk of new-onset postoperative GERD after SG [3-5, 13]. However, research evaluating the endoscopic prevalence of EO and BO after SG are scarce [3-5]. To our knowledge, this is the first study that explores factors that may influence the development of EO and BO in SG patients. Poor outcome of bariatric surgery has been found to be associated with a higher prevalence of de novo $\mathrm{BO}$ in a longterm observation.

The recent metanalysis showed that the range of GERD prevalence estimates in Europe was as high as between $8.8 \%$ and $25.9 \%$ for the general population [14]. Furthermore, obese individuals have at least 2 -fold increased risk of reflux disease [15] because greater incidence of hiatal hernia and higher intra-abdominal pressure seem to have a substantial impact on the development of GERD [16]. Therefore, bariatric surgeries should primarily have a protective effect on reflux symptoms in obese patients $[9,10]$. Nonetheless, SG itself may promote gastroesophageal reflux due to several contributory factors. The natural anatomical relationship between the lower oesophageal sphincter (LOS), the diaphragm and the angle of His supported by gastric and oesophageal-phrenic ligaments, which act as an anti-reflux system, is disrupted during the surgery [5]. The reduced gastric volume leads to the higher intra-gastric pressure, which disturbs the gastroesophageal pressure gradient. In addition, poor surgical technique may lead to twisting or kinking of the stomach tube, which also influences outcomes [17].

The prevalence of GERD following SG has been proven to be prominent [3-5]. However, majority of research studying the relationship between SG and GERD is based on the clinical evaluation of reflux symptoms rather than endoscopic findings [3, 4]. Data regarding endoscopic prevalence of EO are limited $[4,5]$. Yeung et al. in their metanalysis reported the new-onset oesophagitis rate at $23 \%$ in all studies, but $20 \%$ in the subgroup of long-term studies. Similar results were presented in another study of 110 patients who underwent endoscopy during long-term follow-up post SG, where there was a significant increase in incidence and severity of EO at OGD [5]. Our findings indicate a new-onset EO rate at $30 \%$, which is somewhat higher than in the aforementioned studies.

Interestingly, de novo BO was reported in $27 \%$ of our cohort, which is again higher than those reported in other studies, where the incidence rate ranged between 6 and 17\% [4,5, 15]. Although GERD is a key risk factor for the development of $\mathrm{BO}$, it has been postulated that metabolic syndrome along with central obesity plays an independent role in the development of $\mathrm{BO}$, irrespective of the presence of reflux disease $[8,17]$. Visceral adiposity that leads to elevated level proinflammatory cytokines such as C-reactive protein, interleukin- 6 , and tumour necrosis factor- $\alpha$ creates a state of systemic inflammation that has been associated with an increased risk of BO and oesophageal adenocarcinoma [16]. In our study SG patients who developed BO had considerably higher BMI during the long-term follow up than those with EO or normal gastroesophageal junction at OGD. Our findings indicate that patients with poor bariatric outcome or those with weight regain may be at particular risk of $\mathrm{BO}$, a known precursor lesion for oesophageal adenocarcinoma. However, our sample contains a relatively small number of patients, and further research on a larger group is needed to estimate the prevalence and factors contributing to the development of $\mathrm{BO}$ after sleeve gastrectomy.

Several studies suggest that OGD should be a part of the routine follow-up investigations for SG patients $[5,6]$. The results of our research, in which a significant proportion of SG patients developed de novo EO or BO, support this recommendation. Furthermore, SG patients with poor weight control after surgery should be considered at particularly high risk of development of $\mathrm{BO}$ due to the increased prevalence of reflux disease post SG and persistently high BMI being the 2 independent contributing factors.

Our study has several limitations. First, this study is based on a limited number of patients, which may be too small to draw any final conclusions about the prevalence and additional risk factors of BO. However, a larger group is problematic due to the longitudinal construction of the study. Second, no dietary or medications related causes of GERD have been analysed. Finally, no control group was included in the design of the study. 


\section{Conclusions}

The prevalence of $\mathrm{BO}$ in SG patients is considerable. The risk of $\mathrm{BO}$ increases in patients with high end BMI after SG. Endoscopic surveillance should be considered as part of the follow-up, especially in patients with higher overall risk of $\mathrm{BO}$.

\section{Conflict of interest}

The authors declare no conflict of interest.

\section{References}

1. Angrisani L, Santonicola A, lovino P, et al. Bariatric surgery and endoluminal procedures: IFSO Worldwide Survey 2014. Obes Surg 2017; 27: 2279-89.

2. Berger ER, Huffman KM, Fraker T, et al. Prevalence and risk factors for bariatric surgery readmissions: findings from 130,007 admissions in the metabolic and bariatric surgery accreditation and quality improvement program. Ann Surg 2018; 267: 122-31.

3. Salminen P, Helmiö M, Ovaska J, et al. Effect of laparoscopic sleeve gastrectomy vs laparoscopic Roux-en-Y gastric bypass on weight loss at 5 years among patients with morbid obesity: the SLEEVEPASS randomized clinical trial. JAMA 2018; 319: 241-54.

4. Yeung KTD, Penney N, Ashrafian L, et al. Does sleeve gastrectomy expose the distal esophagus to severe reflux? A systematic review and meta-analysis. Ann Surg 2020; 271: 257-65.

5. Genco A, Soricelli E, Casella G, et al. Gastroesophageal reflux disease and Barrett's esophagus after laparoscopic sleeve gastrectomy: a possible, underestimated long-term complication. Surg Obes Relat Dis 2017; 13: 568-74.

6. Nadaleto BF, Herbella FA, Patti MG. Gastroesophageal reflux disease in the obese: pathophysiology and treatment. Surgery 2016; 159: 475-86.

7. Chang P, Friedenberg F. Obesity and GERD. Gastroenterol Clin North Am 2014; 43: 161-73.

8. Leggett CL, Nelsen EM, Tian J, et al. Metabolic syndrome as a risk factor for Barrett esophagus: a population-based case-control study. Mayo Clin Proc 2013; 88: 157-65.

9. Khan A, Kim A, Sanossian C, Francois F. Impact of obesity treat ment on gastroesophageal reflux disease. World I Gastroenterol 2016; 22: 1627-38.

10. Chiu S, Birch DW, Shi X, et al. Effect of sleeve gastrectomy on gastroesophageal reflux disease: a systematic review. Surg Obes Relat Dis 2011; 7: 510-5.

11. Levine DS. Management of dysplasia in the columnar-lined esophagus. Gastroenterol Clin North Am 1997; 26: 613-34.

12. Neagoe R, Muresan M, Timofte D, et al. Long-term outcomes of laparoscopic sleeve gastrectomy - a single-center prospective observational study. Videosurgery Miniinv 2019; 14: 242-8.

13. Gemici E, Kones O, Seyit H, et al. Outcomes of laparoscopic sleeve gastrectomy by means of esophageal manometry and pH-metry, before and after surgery. Videosurgery Miniinv 2020; 15: $129-35$.
14. El-Serag HB, Sweet S, Winchester CC, Dent J. Update on the epidemiology of gastro-oesophageal reflux disease: a systematic review. Gut 2014; 63: 871-80.

15. Felsenreich DM, Kefurt R, Schermann M, et al. Reflux, sleeve dilation, and Barrett's esophagus after laparoscopic sleeve gastrectomy: long-term follow-up. Obes Surg 2017; 27: 3092-101.

16. Ryan AM, Healy LA, Power DG, et al. Barrett esophagus: prevalence of central adiposity, metabolic syndrome, and a proinflammatory state. Ann Surg 2008; 247: 909-15.

17. Mion F, Tolone S, Garros A, et al. High-resolution impedance manometry after sleeve gastrectomy: increased intragastric pressure and reflux are frequent events. Obes Surg 2016; 26: 2449-56.

Received: 19.09.2020, accepted: 2.05.2021 